\title{
Wide-field X-ray Optics
}

\author{
René Hudec ${ }^{1}$, Adolf Inneman ${ }^{2}$, Ladislav Pina ${ }^{3}$, Petr $\check{R} e h a ́ k^{4}$, \\ Paul Gorenstein ${ }^{5}$
}

1 Astronomical Institute, Academy of Sciences of Czech Republic, 25165 Ondřejov, Czech Republic

2 KOMA, Composite Materials, K lesu 965, CZ-142 00 Praha 4, Czech Republic

3 Faculty of Nuclear Engineering, Czech Technical University, Břehová 7, CZ-115 19 Praha 1, Czech Republic

4 Development-Optical Workshop of the Academy of Sciences of Czech Republic, Skálova 89, CZ-511 01 Turnov, Czech Republic

5 Harvard-Smithsonian Center for Astrophysics, 60 Garden Street, Cambridge, MA 02138, USA

Abstract: Classical X-ray mirror optics usually has a limited Field of View (FoV) of $\sim 1^{\circ}$. Wide-field imaging can be achieved by lobster-eye type reflecting X-ray optics. We summarise several different approaches and suggest an innovative technology for the production of $\mathrm{X}$-ray reflecting flats and cells necessary to develop one- or two-dimensional wide-field X-ray optics. The technology is based on double-sided, replicated reflecting foils produced by electroforming and $\mathrm{CF} /$ composite technologies.

\section{Introduction}

The use of double reflecting mirrors for focusing X-rays was suggested by Hans Wolter (1952). The grazing incidence mirrors of the Wolter 1 type appear to be most advantageous for space astronomy applications. However, they have a limited FoV of order $1^{\circ}$. Lobster-eye X-ray optics, suggested more than 20 years later by Schmidt and Angel (Schmidt 1975, Angel 1979), represents an alternative to achieve large FoV. However, in this case the production poses difficulties. No lobster-eye $x$-ray objectives have been constructed to date. We outline possible geometries as well as possible production methods. A very promising new method is based on replica technology. The idea is to create a perfect copy of a negatively shaped master. We have carried out numerous tests and shown that this can even be done several times for particular masters, and that the degradation of shape and/or surface quality is within acceptable limits (Hudec et al. 1988).

The replication process begins with the production (with optical grinding and polishing) of high quality glass or glass ceramic masters (of negative shape). Surface smoothness of the masters can be enhanced using either superpolishing or lacquer coating. The final mirrors are produced by using electrodeposition (galvanoplastics) of a nickel layer on a polished glass master which is then removed. In some cases, the electroformed nickel deposit will be reinforced by composite material (external epoxy-carbon fibre layer). The material of the reflecting surfaces is electroformed nickel with good reflectivity (e.g. $60 \%$ at 0.83 
$\mathrm{nm}$ and $1^{\circ}$ incidence angle). An application of supplementary reflective film (e.g. gold coating) is also possible. For flat mirrors, float glass can be used instead of polished masters. Recently, we considered different high-quality float glasses with surface micro-roughness scales below $1 \mathrm{~nm}$.

\section{The lobster-eye X-ray optics}

The X-ray optics of the lobster-eye geometry offers an excellent opportunity to achieve very large fields of view for energies between 0.1 and $10 \mathrm{keV}$. Two somewhat different systems have been suggested.

\subsection{One dimensional systems}

A one dimensional lobster-eye geometry was originally suggested by Schmidt (1975), based upon flat reflectors. The device consists of a set of flat reflecting surfaces. The plane reflectors are arranged in an uniform radial pattern around the perimeter of a cylinder of radius $R$. X-rays from a given direction are focussed to a line on the surface of a cylinder of radius $R / 2$. The azimuthal angle is determined directly from the centroid of the focused image. At a glancing incidence angle for X-rays of wavelength $1 \mathrm{~nm}$ (and longer), this device can be used to focus a sizable fraction of an intercepted beam of $\mathrm{X}$-rays incident in parallel. Focussing is not perfect and the image size is finite. However, a one dimensional focusing device offers a wide FoV, up to maximum of $2 \pi$ with a coded aperture. It appears possible to achieve an angular resolution of $0: 1$ or better.

Two such systems in sequence, to form a double-focusing device, should offer a FoV of up to 1000 square degrees at moderate angular resolution. We propose the use of such devices in X-ray astronomy to survey or monitor the sky.

Innovative very wide-field $\mathrm{X}$-ray telescopes have been suggested based on these optical elements but have sofar not been flown in space. One of the proposals is the All Sky Supernova and Transient Explorer (ASTRE) (Gorenstein, 1987). This proposal also includes a cylindrical coded aperture outside of the reflectors which provide angular resolution along the cylinder axis. The coded aperture contains circumferential open slits (1 mm wide) in a pseudo-random pattern. The line image is modulated along its length by the coded aperture. The image is cross-correlated with the coded aperture to determine the polar angle of one or more sources. The field of view of this system can, in principle, be as large as $360^{\circ}$ in the azimuthal direction and nearly $90 \%$ of the solid angle in the polar direction.

To create this mirror system, a development of double-sided flats is necessary, while recent $\mathrm{X}$-ray foils are usually one-sided. There is potential for extending the wide field imaging system to higher energy with multilayer coatings analog to those described by Joensen et al. (1994) for flat reflectors in the KirkpatrickBaez geometry. These coatings excert great stress on the substrate. The system must meet severe weight limitations and so the new development of double-sided 
flats reinforced by composite material (to keep weight minimal while preserving mechanical stability) must be initiated.

This is the goal of the new development in which innovative technologies for double-sided flats are tested. The basis of the sandwich-type construction of the X-ray flats is an electroformed nickel layer which is deposited on plates of float glass. The nickel-coated plates of float glass are connected by means of carbon/fibre composite material and after hardening the set of connected plates will be cut/ground off. Subsequently, the plates of glass and the produced composite sandwiches with double-sided nickel mirror foils and inner composite reinforcement will be separated. These foils are lacquered on both sides so that the surface microroughness can reach values under $1 \mathrm{~nm}$. The foils will be covered by a thin gold layer at the final stage.

\subsection{Two dimensional systems}

The idea of two dimensional lobster-eye type wide-field X-ray optics was first mentioned by Angel (1979). The lobster-eye optical grazing incidence X-ray objective consists of numerous tiny square cells located on the sphere and is similar to the reflective eyes of macruran crustaceans, such as lobsters. The FoV can be made as large as desired, and it is possible to achieve high efficiency for photon energies up to $10 \mathrm{keV}$. Spatial resolution of a few seconds of arc over the full field of view is possible, in principle, if very small reflecting cells can be fabricated.

This idea has not been developed further because of difficulties in the production of numerous polished square cells of very small size (about $1 \times 1 \mathrm{~mm}$ or less at lengths of order of tens of $\mathrm{mm}$ ). On the other hand, the very wide field imaging of the sky in $\mathrm{X}$-rays would have very important consequences for a number of applications in X-ray and $\gamma$-ray astronomy.

This demand can be satisfied by electroformed replication and first test cells have already been successfully fabricated. This innovative technology is now under intensified study and a proposal was submitted to the Grant Agency of the Czech Republic. The recent approach is based on electroforming and composite material technology to produce identical triangular segments with square cells to be aligned in quadrants on a sphere. First test cells of $2 \times 2 \mathrm{~mm}$ were successfully produced, indicating that electroforming can be a valuable tool in this very complicated and not yet solved technical problem.

\section{Discussion}

Without doubt, the use of very wide-field X-ray imaging systems would have a significant impact in many areas of $\mathrm{X}$-ray and $\boldsymbol{\gamma}$-ray astrophysics. The production of corresponding optical elements can be achieved by methods of electroforming and composite replication as an alternative to other methods. Results obtained with the developed new technologies for production of large area and high quality one-sided X-ray foils are very promising and together with composite material 
technologies represent important input for the development of double-sided flats needed for lobster-eye geometries of X-ray optics.

Acknowledgements: The development of double-sided X-ray reflecting foils is supported by a grant within the US-Czech Science and Technology Program, No. 93037 .

\section{References}

Angel J.R.P., 1979, ApJ 233, 364

Gorenstein P., 1987, in Variability of Galactic and Extragalactic X-ray Sources, A. Treves (ed.), Associazione per L'Avanzamento dell'Astronomia, Milano-Bologna

Hudec R., Valniček B., 1984, Adv. Space Res. 3, No. 10-12, 545

Hudec R., Valniček B., 1986, SPIE Proc. 597, 111

Hudec R., Valniček B., Aschenbach B., Braeuninger H., Burkert W., 1988, Appl. Optics 27,1453

Hudec R., Valniček B., Svátek L., Landa V., 1989, Proc. of the Conference X-Ray Instrumentation in Medicine and Biology, Plasma Physics, Astrophysics and Synchrotron Radiation, Paris, 24-28 April 1989

Joensen K. D., Gorenstein P., Wood J., Christensen F. E., Høgh $\varnothing$ j P., 1994, SPIE 2279, (in press)

Schmidt W.K.H., 1975, Nucl. Instr. and Methods 127, 285

Wolter H., 1952, Ann. Phys. 10, 94 